\title{
Electrically induced breakdown of the quantum Hall effect at different Hall bar widths: Visualizing the edge- and bulk-dominated regimes within a quantum Hall plateau
}

\author{
P. Haremski®, M. Mausser, A. Gauß๑, K. von Klitzing, and J. Weis ๑* \\ Max-Planck-Institut für Festkörperforschung, Heisenbergstrasse 1, D-70569 Stuttgart, Germany
}

(Received 11 September 2020; accepted 5 November 2020; published 24 November 2020)

\begin{abstract}
We present systematic investigations of the electrically induced breakdown of the integer quantum Hall effect (QHE) in (Al,Ga)As-based Hall bars of different widths ranging from 7 to $70 \mu \mathrm{m}$. It is striking that, for the narrow Hall bars, the threshold values for the applied voltage that induces the breakdown of the longitudinal zero-resistance state differ appreciably between the upper and lower sides of a quantum Hall (QH) plateau. When moving from the low magnetic field side of the QH plateau to higher magnetic fields, the threshold rises in a strongly superlinear manner until—at the expected integer value for the Landau level (LL) filling factor in the bulk of the two-dimensional electron system (2DES) - the threshold value abruptly drops to low values for the rest of the plateau. With increasing widths of the Hall bars, the zero-resistance state extends slightly further to higher magnetic field values. The threshold values on the low-magnetic field side are almost independent of the Hall bar width, whereas the threshold values on the high magnetic field side scale linearly with the Hall bar width. These observations correspond perfectly with the microscope picture of the QHE where the biased current flows in a dissipationless manner in electrically incompressible regions of the 2DES of locally the same LL filling factor, driven by the respective drop of the Hall voltage over the width of these incompressible regions. Owing to the self-consistent evolution of the electrically incompressible/compressible landscape within the 2DES as a function of magnetic field, a transition from an edge- to a bulk-dominated QH regime is described within a $\mathrm{QH}$ plateau. This microscopic picture was derived from sophisticated, long-lasting scanning probe experiments measuring Hall potential profiles on typically $15-\mu \mathrm{m}$-wide Hall bars. Conversely, performing systematic electrically induced breakdown measurements like those presented here would allow us to identify the presence of edge- and bulk-dominated regimes of the QHE in a wider variety of samples.
\end{abstract}

DOI: 10.1103/PhysRevB.102.205306

\section{INTRODUCTION}

Systematic scanning force microscopy experiments [1-8] on narrow $(\mathrm{Al}, \mathrm{Ga}) \mathrm{As}$ Hall bars have yielded a microscopic picture of the integer quantum Hall effect (QHE) [9] that is contradictory to a current-carrying edge-state picture $[10,11]$. The key element of the microscopic picture is the formation of an electrically compressible and incompressible landscape within the two-dimensional electron system (2DES). In the quantum Hall $(\mathrm{QH})$ regime, the Hall voltage drops in the Hall bar cross section over incompressible regions of the sameinteger-valued Landau level (LL) filling factor. This leads to a dissipationless Hall current flow within these incompressible regions along the Hall bar where all occupied states in the LLs contribute locally. Along a $\mathrm{QH}$ plateau, there is a distinction between the edge-dominated regime on the low magnetic field side of the Hall resistance plateau and the bulk-dominated

\footnotetext{
*Corresponding author: j.weis@fkf.mpg.de
}

Published by the American Physical Society under the terms of the Creative Commons Attribution 4.0 International license. Further distribution of this work must maintain attribution to the author(s) and the published article's title, journal citation, and DOI. Open access publication funded by the Max Planck Society. regime on the high magnetic field side. On the low magnetic field side, pronounced incompressible strips are present at the 2DES edges, whereas the 2DES bulk is compressible. On the high magnetic field side, a connected incompressible 2DES bulk exists. With rising magnetic field, the evolution of Hall potential profiles shows a smooth transition from the edge- to the bulk-dominated regime within a $\mathrm{QH}$ plateau. Beyond the plateau, the 2DES is mostly compressible, and the Hall potential drop appears-as expected for a Drude-like conductor-over the entire Hall bar width. The current flow is dissipative.

This interpretation is based on theoretical works by Chklovskii et al. $[12,13]$ and, subsequently, the self-consistent screening theory of Gerhardts and colleagues [14-16], where the electron depletion region along the 2DES edges is characterized by the formation of incompressible and compressible strips along the edges. Especially while following a $\mathrm{QH}$ plateau from the low to high magnetic field, the innermost incompressible strips at opposite edges within a Hall bar cross section widens and shifts with rising magnetic field towards the Hall bar center, merging into an incompressible 2DES bulk. Using the LL filling-factor-dependent magnetoconductivity tensor relation between local (nonequilibrium) current density and electrical field, the self-consistent approach allows us to calculate Hall resistance curves with QH plateaus as well as the related Hall potential profiles [17-21]. The 
evolution of the current distribution and therefore the Hall potential profiles along a $\mathrm{QH}$ plateau is in good agreement with experimental findings [17]. As a result of these works, it can be stated that, owing to the electron depletion at the edges of the 2DES, a constant quantized Hall resistance can exist without disorder for the finite range in the magnetic field. Localized electronic states due to disorder are usually assumed to cause a $\mathrm{QH}$ plateau.

Moreover, theoretical work by Gerhardts et al. [17,22] for bias voltage approaching the breakdown and corresponding scanning probe experiments by Panos et al. [8] show for the edge-dominated regime a rising asymmetry in the Hall potential drop between both edges and therefore in the current distribution. At low bias, the Hall voltage drop and therefore the biased current are symmetrically distributed to both edges flowing in the same direction. With rising bias, the Hall field becomes strongly enhanced locally in the incompressible strip of one edge, whereas the width of the incompressible strip at the opposite edge goes to zero [8]. In the bulk-dominated $\mathrm{QH}$ regime, compressible droplets are embedded in the incompressible bulk owing to inhomogeneities in the electron density [20]. This is where the Hall potential drop localizes with rising bias rather abruptly over a narrow incompressible region between compressible droplets in the Hall bar cross section $[8,23,24]$. The change in an incompressible 2D matrix with compressible droplets at rising Hall field towards more compressibility was simulated numerically in 1997 [25].

The evolutions in the edge- and bulk-dominated regimes towards breakdown are both due to the self-consistent change in the incompressible/compressible landscape with rising Hall voltage. In both cases, this leads to a localized enhancement of the electrical Hall field. Although a comprehensive theory of the electrically induced breakdown has yet to be developed, most breakdown mechanisms discussed in the literature are based on a strong (nonequilibrium) electrical field acting as a trigger, e.g., Joule (Ohmic) heating [26-29], bootstrapping for hot electrons, followed by nonlocal avalanche heating [30-32], intra-LL scattering in a disordered 2DES with spontaneous phonon emission leading to delocalized electron hopping [33,34], quasielastic inter-LL (Zener) tunneling (QUILLS) [35], or impurity-mediated resonant inter-/intra-LL tunneling [36-38].

The electrically induced breakdown measurements reported in the literature use the biased current as the knob parameter. This is a natural approach because applying the QHE as a resistance standard in metrology requires a high current level. On wide Hall bars, the characteristics at midplateau yield the highest critical current value. At the critical current level, the longitudinal resistance changes abruptly in a steplike manner, and hysteresislike and unstable behavior has been observed in increasing and decreasing bias currents around the critical value $[26,39]$. Steplike fluctuations in time are observed by taking traces of the longitudinal voltage versus magnetic field close to the breakdown bias [40,41]. To gain insight into the breakdown mechanism, noise measurements were recently proposed again and used as a tool $[32,42,43]$.

Some works have systematically varied the Hall bar width in electrically induced breakdown investigations (Kawaji et al. [44,45], Balaban et al. [46], Boisen et al. [47], and Oto et al. [48]). As reviewed by Nachtwei [31] in 1999, the critical current level scales linearly or sublinearly with the Hall bar width, depending on the investigated range for the Hall bar width and electron mobility of the 2DES. In Hall bars where the edges are defined by gate electrodes, edge effects were excluded as the origins of the sublinear breakdown behavior because the maximum critical current value was not affected by the applied gate voltage [49]. However, looking at Fig. 2 in Ref. [49], we see that the breakdown behavior along the Hall plateau has changed: the stronger the depletion is, the higher the threshold is on the low magnetic field side of the QH plateau. By comparing magnetocapacitance measurements, the strength of electron density fluctuations (bulk inhomogeneity) of the 2DES seems to be a better parameter than electron mobility for correlation with linear or sublinear breakdown behavior on Hall bar width [31,48]. Mani and Anderson [50] showed as early as 1989 that rising long-range inhomogeneities lower the breakdown threshold.

Artificially penetrating the 2DES bulk in wide Hall bars by means of holes ("antidots") lowers the critical current threshold. This effect is more dramatic in aperiodic antidot arrays than in periodic ones, which hints at a mechanism of dissipation in these samples based on avalanche electron heating and inelastic scattering [51]. The expansion of hot spots at the current-carrying contacts into the Hall bar-nicely imaged by cyclotron photon emission [30,52] - is relevant when the distance to potential probing contacts is too short $[53,54]$. As the electrostatic environment determines the self-consistent evolution of the compressible/incompressible landscape, the presence of close-by electrodes is crucial and might yield a bias polarity dependence on the breakdown behavior (for instance, strongly pronounced in Si-metal-oxide-semiconductor field-effect transistor devices [53]).

Systematic breakdown measurements showing pronounced differences in the behavior between the upper and lower $\mathrm{QH}$ plateau sides have not been discussed in the literature. As stated above, there have been hints at this effect, such as in Fig. 2 in Ref. [49]. In this work, we have examined whether the edge- and bulk-dominated QHE regimes can also be identified in magnetotransport measurements by approaching the electrically induced breakdown of the QHE. Owing to the scanning range, the previous scanning probe experiments were limited to a maximum Hall bar width of $15 \mu \mathrm{m}$. Thus, we have performed systematic electrical magnetoresistance investigations of Hall bars with widths ranging from 7 to $70 \mu \mathrm{m}$ to study the influence of the width on the edge- and the bulk-dominated regimes. Note that we use the DC voltage applied to the Hall bar as the knob parameter because this allows us to keep the compressible/incompressible landscape inside the 2DES from changing abruptly in order to maintain an enforced current level.

\section{SAMPLES AND SETUP FOR ELECTRICAL CHARACTERIZATION MEASUREMENTS}

The samples used in this work are based on an $\mathrm{Al}_{x} \mathrm{Ga}_{1-x} \mathrm{As} / \mathrm{GaAs}$ heterostructure [55] $(x=0.33)$ grown by molecular beam epitaxy. The 2DES is obtained at the $(\mathrm{Al}, \mathrm{Ga}) \mathrm{As} / \mathrm{GaAs}$ heterojunction interface $70 \mathrm{~nm}$ below the surface. The electron concentration of the 2DES is $n=4.5 \times 10^{15} \mathrm{~m}^{-2}$, and the mobility is $\mu=75 \mathrm{~m}^{2} / \mathrm{Vs}$ at 


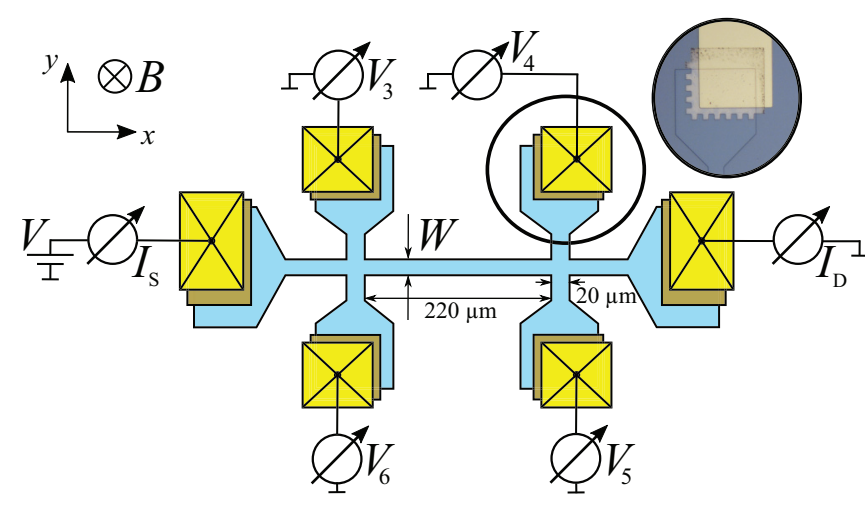

FIG. 1. Sketch of the Hall bar layout and the respective electrical setup to perform DC magnetotransport measurements. The width varies as $W=\{7,14,20,33,70\} \mu \mathrm{m}$. An optical microscopic image of the Ohmic contact encircled in the Hall bar sketch is shown on the top right.

temperature $T=1.3 \mathrm{~K}$. The heterostructure and 2DES parameters are close to those used for our Hall potential profile measurements.

The Hall bar layout and the electrical measurement setup are shown in Fig. 1. We used optical lithography and wet etching to pattern the heterostructure into mesas containing the 2DES. Ohmic contacts were added by alloying $\mathrm{Au} / \mathrm{Ge} / \mathrm{Ni}$ pads into the heterostructure mesa to contact the 2DES [56]. The meandering borderline of the Ohmic contact suppresses the anisotropy of the contact resistance [7,56]. In the final process step, $\mathrm{Cr} / \mathrm{Au}$ pads are evaporated on the sample. The Hall bars differ only in regard to width $W$, whereas the remaining geometry parameters are identical. As the depletion region of the electron density profile at the edges is responsible for the edge-dominated QHE and has a width of about $1 \mu \mathrm{m}$ in such samples [9], the Hall bar width is chosen to be $W=\{7,14,20,33,70\} \mu \mathrm{m}$ so that the contribution of both the edge- and bulk-dominated regimes within the $\mathrm{QH}$ plateau can be investigated. The length of the Hall bar is chosen to be rather long and is $L=220 \mu \mathrm{m}$ between the potential probing contacts.

To examine the width dependence, we perform electrical DC measurements at temperature $T=1.3 \mathrm{~K}$. Hence, a source-drain bias voltage $V$, relative to a common reference, is applied (Fig. 1). The source (S) and drain (D) currents $I_{\mathrm{S}}$ and $I_{\mathrm{D}}$ are measured by current-voltage $(I / V)$ converters [57]. If they are identical, any leakage current can be excluded, and we set $I_{x} \equiv I_{\mathrm{D}}=I_{\mathrm{S}}$. The voltage potentials $V_{3}, V_{4}, V_{5}$, and $V_{6}$ are measured with Keithley 2000 voltmeters in regard to a common reference [58]. Every contact potential is measured separately relative to the reference ground in order to obtain information on how the potentials change relative to each other and along both Hall bar edges relative to the source and drain. By calculating the voltage differences, one obtains the Hall voltages $V_{y}=V_{6}-V_{3}$ and $V_{y}=V_{5}-V_{4}$, respectively, as well as the longitudinal voltage drops $V_{x}=V_{3}-V_{4}$ and $V_{x}=V_{6}-V_{5}$, respectively. Dividing the voltages by the measured current yields the Hall resistance $R_{x y}=V_{y} / I_{x}$ and the longitudinal resistance $R_{x x}=V_{x} / I_{x}$.

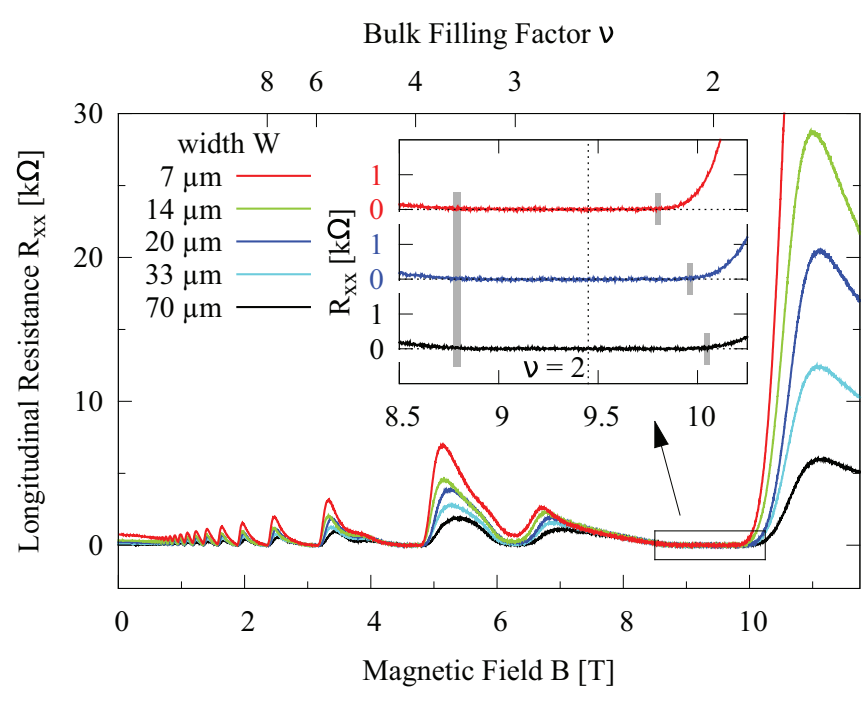

FIG. 2. Longitudinal resistance $R_{x x}$ vs magnetic field $B$ for several Hall bar widths $W$. Inset: Zoom of $R_{x x}$ around filling factor $v=2$ for three different widths. The curves are shifted vertically for better comparison. To guide the eye, the initial and final magnetic field values of zero resistance are marked by gray bars (determined by the same resistance values deviating from zero). Whereas the low magnetic field side does not deviate, the high magnetic field side differs clearly for the various widths.

In this paper, we show the results of magnetoresistance and QHE breakdown measurements [59]. During magnetoresistance measurements, a small constant voltage $V$ is applied while the magnet is swept continuously at a low rate [60]. These nonequilibrium eddy currents affect the breakdown behavior because biased current and eddy currents superpose locally, leading to locally enhanced or reduced Hall fields. For QHE breakdown measurements, the magnetic field is changed in a steplike manner and held at every magnetic field value. Meanwhile the applied source-drain voltage is increased from zero to its maximum value $(300 \mathrm{mV})$. After that, the magnet field is changed to the next value, and the applied voltage is increased anew.

\section{MEASUREMENT RESULTS}

Figure 2 shows the longitudinal resistance $R_{x x}$ plotted as a function of magnetic field $B$ for a small applied voltage $V=$ $2 \mathrm{mV}$ and for different widths $W$ of the Hall bar. The positions for integer values of the bulk filling factor $v$ are also marked. These values of $v$ are calculated from the electron density obtained from the Hall curve slope at low magnetic fields. We concentrate here on the broadest and stablest plateau: the one around filling factor $v=2$. A zoom of this plateau for three different Hall bar widths is shown in the inset. The curves are shifted vertically for better comparison, and the initial and final magnetic field values of $R_{x x}=0$ are indicated by gray bars. On the low magnetic field side of the plateau, we can see that the initial magnetic field value of $R_{x x}=0$ is independent of the Hall bar width. In contrast, there are clear differences on the high magnetic field side of $R_{x x}=0$. For wider Hall bars, the longitudinal resistance remains zero for a larger magnetic field range. Hence, an extended zero-resistance state exists. 

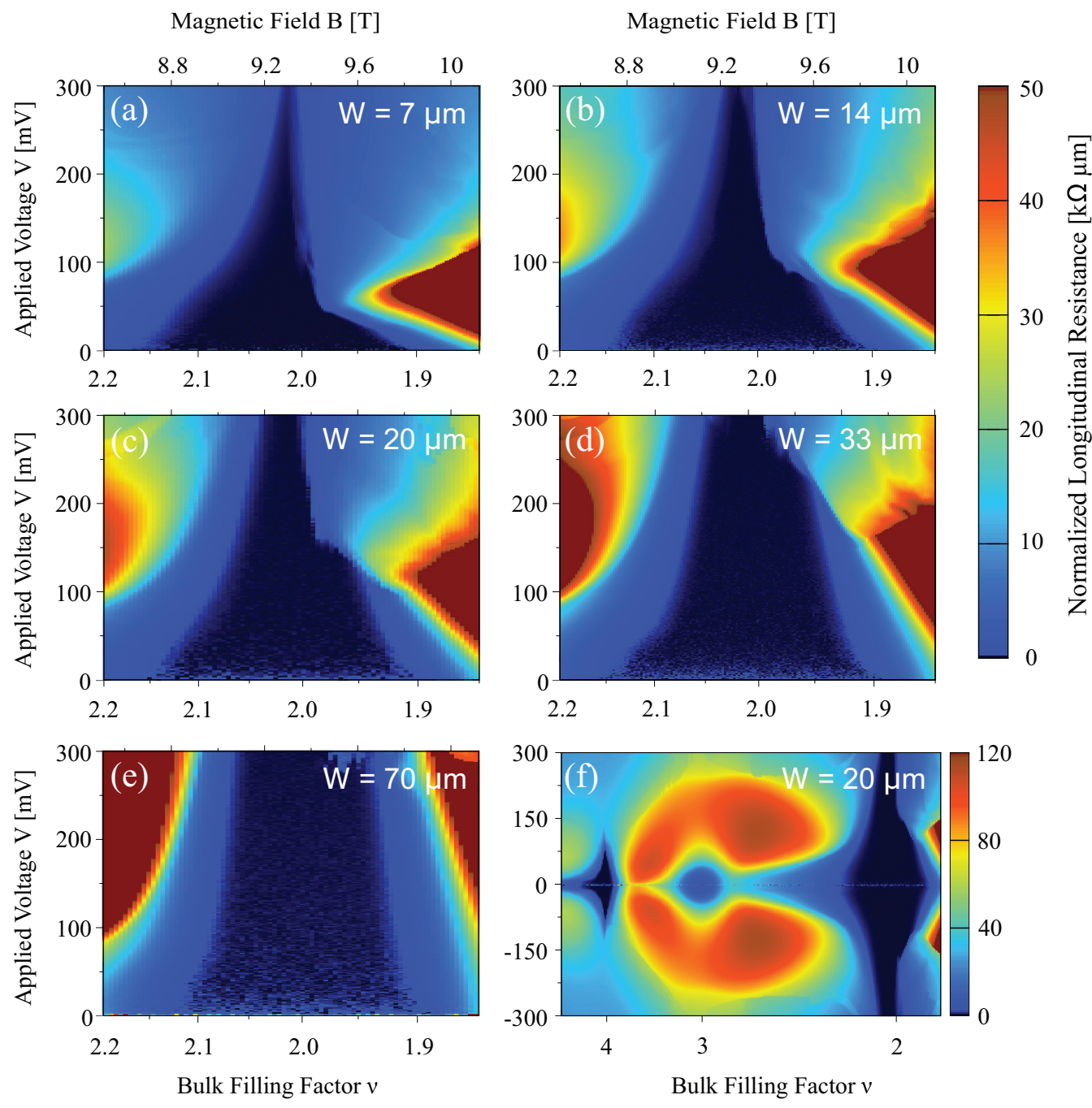

FIG. 3. Color-coded contour plots of normalized longitudinal resistance $\left(W R_{x x}\right)$ around $v=2$ vs bulk filling factor $v$ and applied voltage $V$ for Hall bar widths of (a) $W=7 \mu \mathrm{m}$, (b) $W=14 \mu \mathrm{m}$, (c) $W=20 \mu \mathrm{m}$, (d) $W=33 \mu \mathrm{m}$, and (e) $W=70 \mu \mathrm{m}$. The condition $R_{x x}=0$ is indicated in black. The narrowest Hall bar in (a) shows the largest asymmetry between the low and high magnetic field regimes of the QHE. For wider Hall bars, the high magnetic field regime becomes increasingly stable against the applied voltage, and the asymmetry in the behavior around $v=2$ decreases until it vanishes almost completely in (e). (f) Color-coded $W R_{x x}$ for Hall bar $W=20 \mu \mathrm{m}$ vs magnetic field-ranging from the $\mathrm{QH}$ plateau around $v=4$ to the one around $v=2$-and bias voltage $V$ from negative to positive polarity.

As early as 1987, Kane et al. [61] compared the longitudinal resistance curve at a small bias current for different Hall bar widths and found that the resistance values just before entering the $R_{x x}=0$ state from the low magnetic field side are independent of the Hall bar width, whereas the rise in resistance values beyond the $R_{x x}=0$ state at high magnetic fields shows inverse linear scaling with width. The same is shown by our data in Fig. 2. On the low magnetic field side just before the $\mathrm{QH}$ plateau is reached, a small biased current flows in "filaments" within the depletion region along the 2DES edges, which makes the resistance independent of the Hall bar width, whereas on the high magnetic field side beyond the $\mathrm{QH}$ plateau, the whole 2DES bulk width determines the resistance. We know from scanning probe experiments $[3,4]$ that, before even entering the $\mathrm{QH}$ plateau, most of the biased current can flow inside the innermost incompressible strips along both edges, which are not yet well pronounced. In other words, they are not wide enough and therefore not yet sufficiently isolating to prohibit electron scattering between the compressible edges and the compressible bulk [3,4,9]. Consequently, a fraction of the biased current flows in the compressible bulk, which, however, becomes less and less as we approach the $\mathrm{QH}$ plateau and ultimately vanishes upon entering the plateau.

The data shown in Fig. 2 suggest that an edge-dominated regime exists just before the QH plateau and that a bulkdominated regime exists after the $\mathrm{QH}$ plateau. However, it does not show the evolution along the zero-resistance state/QH plateau. Here we obtain information about the evolution from breakdown measurements for different Hall bar widths [8]. In Fig. 3, the normalized longitudinal resistance $\left(W R_{x x}\right)$ around $v=2$ is shown on a color scale for various Hall bar widths as a function of the magnetic field $B$ /filling factor $v$ and the applied voltage $V$. Note that, owing to the low contact resistances, the bias voltage appears in the $\mathrm{QH}$ regime at nearly full strength as a Hall voltage over the Hall bar cross section. The QHE is stable as long as the 


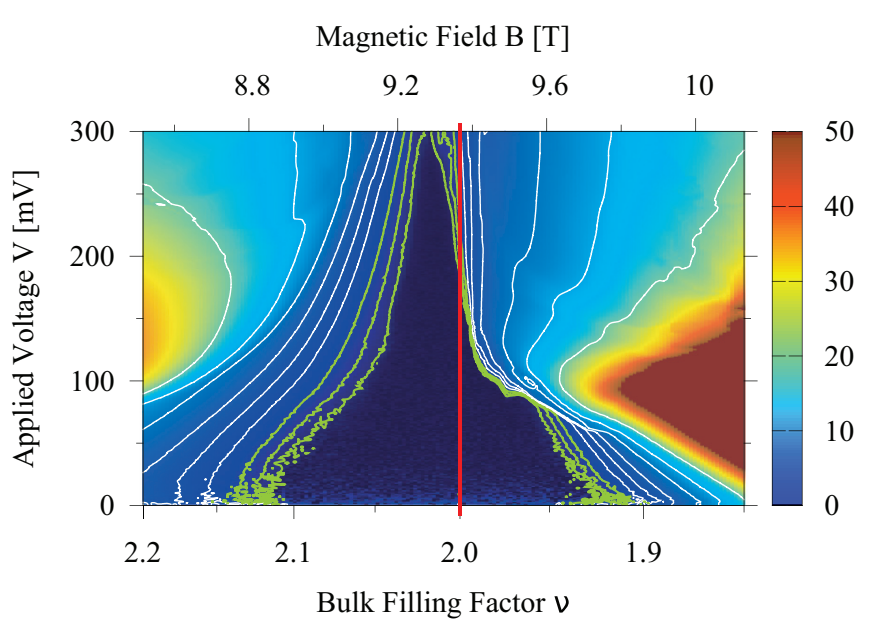

FIG. 4. Normalized longitudinal resistance around $v=2$ for $W=14 \mu \mathrm{m}$. The red line indicates the filling factor $v=2$ determined from the electron density obtained in Hall measurements with an uncertainty of a few percent. The values of the white contour lines from the inside to the outside (in $\mathrm{k} \Omega \mu \mathrm{m}$ ) are $0.5,1,2,5,10,20$. The threshold value for the electrically induced breakdown is chosen to be $0.1 \mathrm{k} \Omega \mu \mathrm{m}$, and two additional contour lines (green) with 0.05 and $0.2 \mathrm{k} \Omega \mu \mathrm{m}$ are added to show a gradient. The asymmetry between the low and high magnetic field regimes of the plateau indicates the edge- and bulk-dominated regimes of the QHE.

longitudinal resistance is zero, i.e., $R_{x x}=0$. In the diagram, this condition is fulfilled for the regions shown in black [62]. For a fixed magnetic field value, the electrically induced breakdown sets in when the longitudinal resistance starts to differ from zero. This means that, in the color-coded contour plots, the breakdown is visible when the color changes from black to blue. By comparing the low and high magnetic field regimes of the QHE (regions shown in black), the narrowest Hall bar in Fig. 3(a) shows the greatest asymmetry around $v=2$, i.e., in the threshold voltage. For wider Hall bars, this asymmetry decreases until it vanishes almost completely, as shown in Fig. 3(e). The QHE in the high magnetic field regime becomes increasingly stable with increasing widths against the applied voltage and extends to slightly higher magnetic field values, which is consistent with the observation of the magnetoresistance measurements in Fig. 2. The observed asymmetry for narrow Hall bars is also pronounced around a filling factor of 4, as shown in Fig. 3(f). Symmetric breakdown behavior was also observed on wide (Al,Ga)As Hall bars for higher filling factors by [63]. In a recent work [64], extended breakdown measurements on $15-\mu \mathrm{m}$-wide InGaAs/InP Hall bars were presented, in which the electron density had been systematically varied. There the differential Hall and longitudinal resistance vs DC bias current and magnetic field was plotted. The pronounced asymmetric behavior shown in Fig. 3 for almost the same Hall bar width of $14 \mu \mathrm{m}$ was not reported.

\section{DISCUSSION}

To see how the results of the breakdown measurements can be interpreted within the microscopic picture of the QHE [9], let us examine Fig. 4, where the normalized longitudinal resistance is plotted for the example of $W=14 \mu \mathrm{m}$.
Furthermore, contour lines for selected values of the normalized longitudinal resistance (listed in the Fig. 4 caption) are added. In the vicinity of the electrically induced breakdown (see the first six contour lines from the inside), the paths of the contour lines are mostly parallel, at least in part. The behavior starts to deviate only for higher values. This means that we can choose a threshold value for the electrically induced breakdown beyond the noise level. We have opted for a threshold value of $0.1 \mathrm{k} \Omega \mu \mathrm{m}$ and added another two contour lines at 0.05 and $0.2 \mathrm{k} \Omega \mu \mathrm{m}$ to see a gradient in the evolution of breakdown with rising bias voltage. These contour lines are highlighted in green and describe the development of the electrically induced breakdown along the $\mathrm{QH}$ plateau. On the low magnetic field side, the normalized longitudinal resistance increases smoothly, whereas on the high magnetic field side, the breakdown occurs rather abruptly and is accompanied by a steep increase in the normalized longitudinal resistance.

Based on the microscopic picture of the QHE, this asymmetric behavior in the breakdown between the low and high magnetic field sides of the plateau indicates the edge- and bulk-dominated regimes of the QHE. Starting on the low magnetic field side of the plateau, we see a strong increase in the breakdown threshold until the integer filling factor (red line) is reached, where an abrupt decrease of the threshold is observable. From the microscopic picture of the $\mathrm{QH}$ effect, we can expect this: With increasing magnetic field, the QHE becomes more stable against the applied voltage/Hall voltage as the innermost incompressible strips at the edges become broader. They move simultaneously from both edges of a Hall bar cross section towards the bulk, and for the integer bulk filling factor, they have merged to a mostly incompressible bulk. This is the point where we see the abrupt decrease of the threshold voltage. Without disorder we would expect the bulk to become incompressible for $v=2$ and then abruptly compressible for slightly lower values of $v$. However, owing to inhomogeneities in the electron density, the incompressible bulk is penetrated by compressible droplets maintaining a connected incompressible bulk for even higher magnetic fields.

Plotting the three threshold contour lines for each Hall bar width leads to Fig. 5(a) and yields a well-founded basis to investigate the width dependence of the QHE by means of the electrically induced breakdown. Below the innermost contour line, the QHE is stable (previously, this region was colored black). By comparing these charts, we can identify three regimes within the $\mathrm{QH}$ plateau, which will be analyzed in the following.

\section{A. Bulk-dominated $\mathrm{QH}$ regime}

We attribute the $\mathrm{QH}$ plateau regime below $v<2$ to the bulk-dominated $\mathrm{QH}$ regime where electron density variations lead to a connected incompressible 2DES with compressible droplets embedded [Fig. 6(d)] [65]. Here we observe the strongest dependence of the breakdown on Hall bar width: the wider the Hall bar is, the higher the threshold voltage is.

In order to analyze its dependence, the applied voltage $V$ is divided by width $W$ for the sake of normalization. Therefore, Fig. 5(a) needs to be scaled only in the $V$ axis. The result is shown in Fig. 5(b). In this regime, the contour lines overlap 


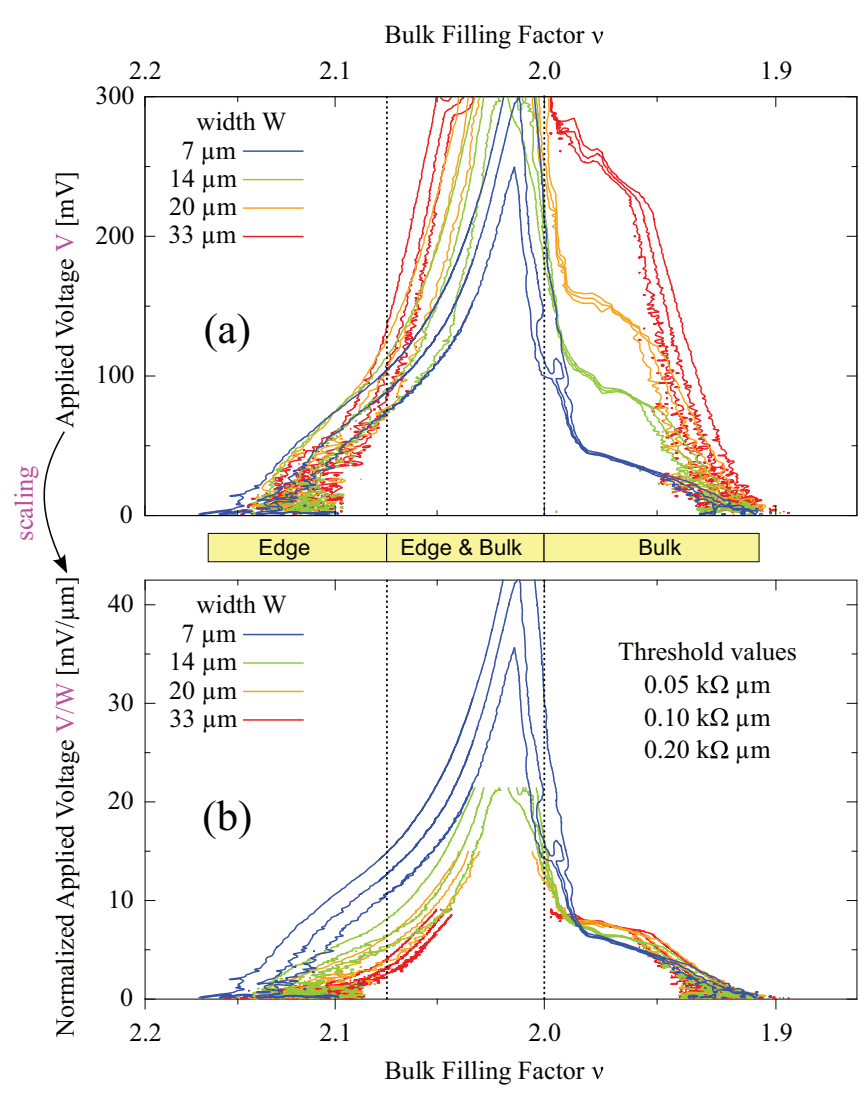

FIG. 5. Threshold contour lines of the electrically induced breakdown in the normalized longitudinal resistance $\left(W R_{x x}\right)$ around $v=2$ for various widths $W$ vs $v$ and (a) the applied voltage $V$ or (b) the normalized applied voltage $V / W$. (a) By comparing the two graphs, we can identify three different regimes within the QH plateau. In the edge-dominated $\mathrm{QH}$ regime, the development of the breakdown threshold is independent of the width. In the mixed edge-bulk QH regime, the width dependence and a strong increase of the threshold voltage are observable. Incompressible edge strips and bulk disorder play a role. In the bulk-dominated $\mathrm{QH}$ regime, strong width dependence is observable. Scaling the voltage by dividing it by $W$ yields the diagram shown in (b). The contour lines in the bulk-dominated regime now match, so the threshold voltage scales linearly with $W$ in this regime.

reasonably well. We can conclude that the threshold voltage in the bulk-dominated regime scales linearly with the Hall bar width in a first approximation.

This result was expected because we know from scanning probe experiments $[8,66]$ that a low applied voltage leads to a widely distributed Hall voltage drop over all incompressible segments within the Hall bar cross section [see Fig. 6(e)]. With increasing applied voltage, the Hall potential drop remains widely spread until a threshold is reached where an abrupt change in the Hall potential profile occurs: A large drop in Hall voltage occurs over a small incompressible segment in the 2DES bulk, and the electric field is locally strongly enhanced, as shown in Fig. 6(f). This situation is a prerequisite for various breakdown mechanisms. As the incompressible bulk becomes broader for wider Hall bars, the Hall voltage can drop over a wider region. Thus, higher voltages can be applied to wider Hall bars before the electrically induced breakdown sets in. Looking at Fig. 4, we see that the distance between contour lines in the regime $v<2$ at breakdown is tiny, which indicates a rather abrupt rise in the longitudinal resistance with voltage bias. That is observed for all Hall bar widths [see Fig. 5(a)].

Looking more carefully at $v<2$ in Fig. 5(b), one realizes that, for the narrow Hall bar, the breakdown occurs at slightly lower $V / W$ values. This might not be surprising because, in the narrowest Hall bar, the depletion widths (about $1 \mu \mathrm{m}$ ) at both edges take a significant fraction of the Hall bar width $W$; that is, the ratio between 2DES bulk width and Hall bar width is diminished.

\section{B. Edge-dominated QH regime}

On the low magnetic field side of the $\mathrm{QH}$ plateau, we expect that pronounced incompressible strips that widen with rising magnetic field in the depletion region along the Hall bar edges would be able to carry the current. We know from scanning probe experiments that, for small applied voltages, the Hall voltage drop is distributed equally over the innermost incompressible strip to both edges in a Hall bar cross section, as sketched in Fig. 6(b). With increasing applied voltage, an asymmetry in the Hall potential drop-and therefore in the current distribution - arises continuously between the left and right edges of the Hall bar [see Fig. 6(c)] [8,66]. This results in a dominant incompressible strip with enhanced electric field on one edge, which makes it possible for breakdown mechanisms such as QUILLS [35] and Joule heating [26-28,30] to occur. On the other edge, i.e., the positive Hall voltage side, the possible Hall potential drop is limited by the energy gap between LLs as the width of the incompressible strip shrinks to zero and electrons from the bulk may relax into the outer edge by phonon and/or photon emission $[67,68]$. As the 2DES bulk is compressible, its width should not matter for the breakdown threshold. Indeed, Fig. 5 shows that the breakdown threshold does not scale with the Hall bar width.

\section{Mixed edge-bulk QH regime}

For narrow Hall bars, the edge-dominated QH regime dominates until $v=2$. For the widest Hall bar, the breakdown threshold starts already at $v \approx 2.09$ to scale with the Hall bar width. Electron density variations in the 2DES bulk not only extend incompressibility to $v<2$ but also lead to incompressibility in the bulk for $v>2$. In this transition regime, both the incompressible edge strips and the bulk disorder are essential, in agreement with the scanning force microscopy measurements by Panos [66]. The Hall potential drop and the current are rearranged over all incompressible segments, thus allowing higher voltages before the electrically induced breakdown sets in. Electrical transport coexists along the edges and is distributed in the bulk. For wider Hall bars, the incompressible bulk in the sample center is broader, which means that a higher fraction of the Hall voltage can drop within the bulk. Even higher voltages can be applied without breaking down the QHE. The transition region depends strongly on sample details and on how the bulk disorder evolves. 
(a)

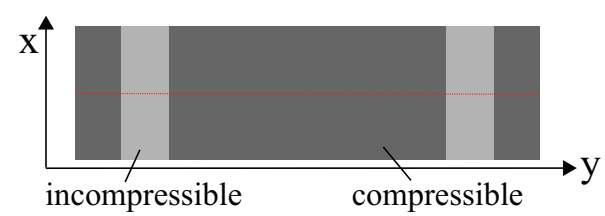

(b)

(c)

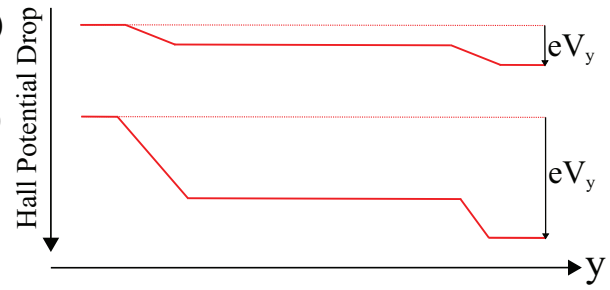

(d)

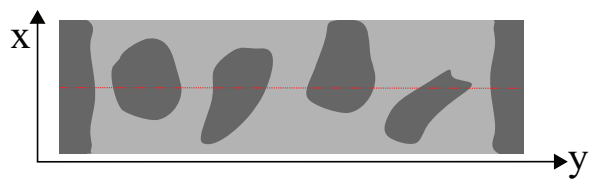

(e)

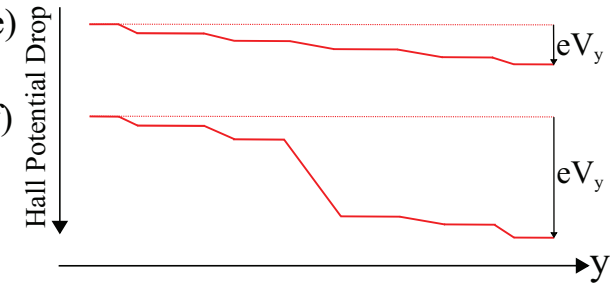

FIG. 6. Sketch of the compressible and incompressible landscapes as well as the respective Hall potential drop over incompressible regions within (a)-(c) the edge-dominated and (d)-(f) the bulk-dominated QH regimes. The Hall potential drop is sketched within a cross section of the sample for (b) and (e) low and (c) and (f) high Hall voltage $V_{y}$. For edge-dominated QHE, with increasing Hall voltage, a dominant incompressible strip with enhanced electric field evolves on one side (shown here on the left). For bulk-dominated QHE, compressible droplets on the micrometer scale are present in the incompressible bulk. For low applied voltage, the Hall voltage drops, which is widely distributed over incompressible sections between compressible droplets. With increasing voltage, the Hall potential profile remains widely distributed until, at a certain high voltage, the Hall potential drop occurs over a narrow incompressible section in the bulk. Here the electric field is locally enhanced, which is a prerequisite for the electrically induced breakdown of the QHE.

\section{CONCLUSION}

In this paper, we have systematically investigated the electrically induced breakdown of the QHE along a QH plateau $(v=2)$ for different Hall bar widths. As is commonly done, we have investigated the existence of a zero-resistance state in longitudinal resistance as an indication of a $\mathrm{QH}$ state. The asymmetric behavior of the breakdown threshold value around $v=2$ for narrow Hall bars confirms the microscopic picture of the QHE based on the self-consistent evolution of the compressible/incompressible landscape inside the 2DES as a function of magnetic field and bias voltage. The edge- and the bulk-dominated $\mathrm{QH}$ regimes are obvious. At intermediate Hall bar widths, a mixed edge-bulk regime is identified at $v>2$, where the bulk contribution becomes dominant with increasing Hall bar widths.

We observe that the magnetic field value that starts the zero-resistance state is independent of the Hall bar width, whereas the magnetic field value that ends the zero-resistance state shifts to higher magnetic field values with increasing Hall bar widths. We conclude that, with increasing 2DES widths, a pronounced incompressible path in 2DES bulk exists along the Hall bar length to even higher magnetic fields, thus allowing dissipationless current flow at small bias voltages. We measured the longitudinal resistance over a length of $220 \mu \mathrm{m}$, but the Hall bar width was varied only from 7 to $70 \mu \mathrm{m}$. Therefore, we cannot state that the inhomogeneity of the 2DES has risen due to the enlarged 2DES area we are probing. However, the chance for more and wider incompressible sections in the Hall bar cross section has risen with increased width.

From the microscopic picture of the QHE, we know that an edge-dominated $\mathrm{QH}$ regime $\left(R_{x x}=0\right.$ and $R_{x y}$ quantized) exists only when a pronounced incompressible strip is present along the edges of the 2DES and in front of alloyed ohmic contacts; that is, the compressible bulk and compressible edge are not shortened by the metallic contacts [9]. Former scanning probe experiments on 2DES embedded in $(\mathrm{Al}, \mathrm{Ga}) \mathrm{As}$ heterostructures have indeed shown that there exists a partial electron depletion along the border between the 2DES and the metal contacts. This allows the innermost incompressible strip along an etched mesa to pass in front of the border between the 2DES and the metal contacts [5,7]. In general, we cannot expect that the width of the incompressible strip along the mesa edge is equal to the one along a metal contact, which would allow different Hall voltage drops before breakdown sets in. From the measurements presented here, we cannot tell where the edge-dominated $\mathrm{QH}$ regime breaks down, i.e., whether it occurs along the etched mesa edges or in front of the metal contacts. This requires investigation of another set of QH samples, where gate electrodes allow the width of the incompressible strips in front of contacts and/or along the mesa edges to be tuned independently.

\section{ACKNOWLEDGMENTS}

The authors thank M. Hauser and W. Dietsche for providing (Al,Ga)As heterostructures, and A. Güth and M. Hagel for sample processing.
[1] K. L. McCormick, M. T. Woodside, M. Huang, M. Wu, P. L. McEuen, C. Duruoz, and J. S. Harris, Scanned potential microscopy of edge and bulk currents in the quantum Hall regime, Phys. Rev. B 59, 4654 (1999). 
[2] P. Weitz, E. Ahlswede, J. Weis, K. v. Klitzing, and K. Eberl, A low-temperature scanning force microscope for investigating buried two-dimensional electron systems under quantum Hall conditions, Appl. Surf. Sci. 157, 349 (2000).

[3] P. Weitz, E. Ahlswede, J. Weis, K. von Klitzing, and K. Eberl, Hall-potential investigations under quantum Hall conditions using scanning force microscopy, Phys. E (Amsterdam, Neth.) 6, 247 (2000).

[4] E. Ahlswede, P. Weitz, J. Weis, K. von Klitzing, and K. Eberl, Hall potential profiles in the quantum Hall regime measured by a scanning force microscope, Phys. B (Amsterdam, Neth.) 298, 562 (2001).

[5] E. Ahlswede, J. Weis, K. v. Klitzing, and K. Eberl, Hall potential distribution in the quantum Hall regime in the vicinity of a potential probe contact, Phys. E (Amsterdam, Neth.) 12, 165 (2002)

[6] J. Weis, Hall potential profiles in quantum Hall samples measured by a low-temperature scanning force microscope, Int. J. Mod. Phys. B 21, 1297 (2007).

[7] F. Dahlem, E. Ahlswede, J. Weis, and K. v. Klitzing, Cryogenic scanning force microscopy of quantum Hall samples: Adiabatic transport originating in anisotropic depletion at contact interfaces, Phys. Rev. B 82, 121305(R) (2010).

[8] K. Panos, R. R. Gerhardts, J. Weis, and K. von Klitzing, Current distribution and Hall potential landscape towards breakdown of the quantum Hall effect: A scanning force microscopy investigation, New J. Phys. 16, 113071 (2014).

[9] J. Weis and K. von Klitzing, Metrology and microscopic picture of the integer quantum Hall effect, Philos. Trans. R. Soc. A 369, 3954 (2011).

[10] B. I. Halperin, Quantized Hall conductance, current-carrying edge states, and the existence of extended states in a twodimensional disordered potential, Phys. Rev. B 25, 2185 (1982).

[11] M. Büttiker, Absence of backscattering in the quantum Hall effect in multiprobe conductors, Phys. Rev. B 38, 9375 (1988).

[12] D. B. Chklovskii, B. I. Shklovskii, and L. I. Glazman, Electrostatics of edge channels, Phys. Rev. B 46, 4026 (1992).

[13] D. B. Chklovskii, K. A. Matveev, and B. I. Shklovskii, Ballistic conductance of interacting electrons in the quantum Hall regime, Phys. Rev. B 47, 12605 (1993).

[14] K. Lier and R. R. Gerhardts, Self-consistent calculations of edge channels in laterally confined two-dimensional electron systems, Phys. Rev. B 50, 7757 (1994).

[15] J. H. Oh and R. R. Gerhardts, Self-consistent Thomas-Fermi calculation of potential and current distributions in a twodimensional Hall bar geometry, Phys. Rev. B 56, 13519 (1997).

[16] A. Siddiki and R. R. Gerhardts, Thomas-Fermi-Poisson theory of screening for laterally confined and unconfined twodimensional electron systems in strong magnetic fields, Phys. Rev. B 68, 125315 (2003).

[17] K. Güven and R. R. Gerhardts, Self-consistent local equilibrium model for density profile and distribution of dissipative currents in a Hall bar under strong magnetic fields, Phys. Rev. B 67, 115327 (2003).

[18] A. Siddiki and R. R. Gerhardts, The interrelation between incompressible strips and quantized Hall plateaus, Int. J. Mod. Phys. B 18, 3541 (2004).

[19] A. Siddiki and R. R. Gerhardts, Incompressible strips in dissipative Hall bars as origin of quantized Hall plateaus, Phys. Rev. B 70, 195335 (2004).
[20] A. Siddiki and R. R. Gerhardts, Range-dependent disorder effects on the plateau-widths calculated within the screening theory of the IQHE, Int. J. Mod. Phys. B 21, 1362 (2007).

[21] R. R. Gerhardts, The effect of screening on current distribution and conductance quantisation in narrow quantum Hall systems, Phys. Status Solidi B 245, 378 (2008).

[22] R. R. Gerhardts, K. Panos, and J. Weis, Current-induced asymmetries of incompressible strips in narrow quantum Hall systems, New J. Phys. 15, 073034 (2013).

[23] R. R. Gerhardts, Current distribution in narrow translationinvariant quantum-Hall-systems with lateral density modulation, New J. Phys. 21, 073007 (2019).

[24] (Al,Ga)As samples are rather clean; that is, electron localization due to a strong short-range impurity disorder does not dominate. The magnetic length at several teslas is small compared to the expected intrinsic length scale for potential fluctuations, leading to electron density variations. Therefore, a self-consistent electron screening theory has to be applied, which justifies the picture of compressible droplets embedded in an incompressible matrix. Even in quantum dots and around antidots of submicron size, concentric compressible/incompressible rings have been identified (originally in [69]).

[25] V. Tsemekhman, K. Tsemekhman, C. Wexler, J. H. Han, and D. J. Thouless, Theory of the breakdown of the quantum Hall effect, Phys. Rev. B 55, R10201 (1997).

[26] G. Ebert, K. v. Klitzing, K. Ploog, and G. Weinmann, Twodimensional magneto-quantum transport on $\mathrm{GaAs}-\mathrm{Al}_{x} \mathrm{Ga}_{1-x} \mathrm{As}$ heterostructures under non-ohmic conditions, J. Phys. C 16, 5441 (1983).

[27] S. Komiyama, T. Takamasu, S. Hiyamizu, and S. Sasa, Breakdown of the quantum Hall effect due to electron heating, Solid State Commun. 54, 479 (1985).

[28] T. Takamasu, S. Komiyama, S. Hiyamizu, and S. Sasa, Effect of finite electric field on the quantum Hall effect, Surf. Sci. 170, 202 (1986).

[29] R. R. Gerhardts, Effect of Joule heating on current-induced asymmetries and breakdown of the quantum Hall effect in narrow Hall bars, Phys. E (Amsterdam, Neth.) 85, 38 (2017).

[30] Y. Kawano and S. Komiyama, Breakdown of the quantized Hall effect in the vicinity of current contacts, Phys. Rev. B 61, 2931 (2000).

[31] G. Nachtwei, Breakdown of the quantum Hall effect, Phys. E (Amsterdam, Neth.) 4, 79 (1999).

[32] K. Chida, T. Hata, T. Arakawa, S. Matsuo, Y. Nishihara, T. Tanaka, T. Ono, and K. Kobayashi, Avalanche electron bunching in a Corbino disk in the quantum Hall effect breakdown regime, Phys. Rev. B 89, 235318 (2014).

[33] P. Streda and K. v. Klitzing, Critical non-dissipative current of quantum Hall regime, J. Phys. C 17, L483 (1984).

[34] P. Streda, Breakdown of the quantum Hall effect, J. Phys. C 19, L155 (1986).

[35] L. Eaves and F. W. Sheard, Size-dependent quantised breakdown of the dissipationless quantum Hall effect in narrow channels, Semicond. Sci. Technol. 1, 346 (1986).

[36] V. L. Pokrovsky, L. P. Pryadko, and A. L. Talapov, Resonant tunneling and breakdown of the quantum Hall effect in strong fields, J. Phys.: Condens. Matter 2, 1583 (1990).

[37] V. N. Nicopoulos and S. A.Trugman, Complex Quantum Scattering and the Breakdown of the Quantum Hall Effect, Phys. Rev. Lett. 65, 779 (1990). 
[38] K. Shizuya, Field-induced breakdown of the quantum Hall effect, Phys. Rev. B 60, 8218 (1999).

[39] M. E. Cage, R. F. Dziuba, B. F. Field, E. R. Williams, S. M. Girvin, A. C. Gossard, D. C. Tsui, and R. J. Wagner, Dissipation and Dynamic Nonlinear Behavior in the Quantum Hall Regime, Phys. Rev. Lett. 51, 1374 (1983).

[40] M. E. Cage, G. M. Reedtz, and C. T. V. Degrift, Quantised dissipative states at breakdown of the quantum Hall effect, Semicond. Sci. Technol. 5, 351 (1990).

[41] M. E. Cage, Dependence of quantized Hall effect breakdown voltage on magnetic field and current, J. Res. Natl. Inst. Stand. Technol. 98, 361 (1993).

[42] J. Schurr, F. Ahlers, and L. Callegaro, Noise and correlation study of quantum Hall devices, IEEE Trans. Instrum. Meas. 62, 1574 (2013).

[43] K. Chida, T. Arakawa, S. Matsuo, Y. Nishihara, T. Tanaka, D. Chiba, T. Ono, T. Hata, K. Kobayashi, and T. Machida, Observation of finite excess noise in the voltage-biased quantum Hall regime as a precursor for breakdown, Phys. Rev. B 87, 155313 (2013).

[44] S. Kawaji, K. Hirakawa, and M. Nagata, Device-width dependence of plateau width in quantum Hall states, Phys. B (Amsterdam, Neth.) 184, 17 (1993).

[45] S. Kawaji, K. Hirakawa, M. Nagata, T. Okamoto, T. Fukase, and T. Goto, Magnetic field dependence of the device-widthdependent breakdown current in the quantum Hall effect, Surf. Sci. 305, 161 (1994).

[46] N. Q. Balaban, U. Meirav, H. Shtrikman, and Y. Levinson, Scaling of the Critical Current in the Quantum Hall Effect: A Probe of Current Distribution, Phys. Rev. Lett. 71, 1443 (1993).

[47] A. Boisen, P. Boggild, A. Kristensen, and P. E. Lindelof, Nonlinear current-voltage characteristics at quantum Hall resistance minima, Phys. Rev. B 50, 1957 (1994).

[48] K. Oto, T. Sanuki, S. Tataoka, K. Murase, and K. Gamo, Two types of breakdown of quantum Hall effect depending on the electron density fluctuation, Phys. E (Amsterdam, Neth.) 12, 173 (2002).

[49] K. Oto and T. Tsubota, Bulk nature of sub-linear width dependent breakdown of quantum Hall effect, Phys. E (Amsterdam, Neth.) 22, 197 (2004).

[50] R. Mani and J. R. Anderson, Inhomogeneous broadening and the breakdown of the quantum Hall effect in GaAs/AlGaAs, Solid State Commun. 72, 949 (1989).

[51] G. Nachtwei, G. Lütjering, D. Weiss, Z. H. Liu, K. von Klitzing, and C. T. Foxon, Breakdown of the quantum Hall effect in periodic and aperiodic antidot arrays, Phys. Rev. B 55, 6731 (1997).

[52] Y. Kawano and S. Komiyama, Spatial distribution of nonequilibrium electrons in quantum Hall devices: Imaging via cyclotron emission, Phys. Rev. B 68, 085328 (2003).

[53] P. C. van Son, G. H. Kruithof, and T. M. Klapwijk, Current contacts and the breakdown of the quantum Hall effect, Phys. Rev. B 42, 11267 (1990).

[54] Y. M. Meziani, C. Chaubet, S. Bonifacie, A. Raymond, W. Poirier, and F. Piquemal, Behavior of the contacts of quantum Hall effect devices at high currents, J. Appl. Phys. 96, 404 (2004).

[55] For the layer sequence, on a 0.5 -mm-thick GaAs substrate, a $50 \times(10 \mathrm{~nm}, 2.8 \mathrm{~nm}) \mathrm{Al}_{x} \mathrm{Ga}_{1-x} \mathrm{As} / \mathrm{GaAs}$ superlattice, a 500-nm GaAs layer, a 20-nm $\mathrm{Al}_{x} \mathrm{Ga}_{1-x}$ As spacer, a 20-nm
$\mathrm{Al}_{x} \mathrm{Ga}_{1-x} \mathrm{As}$ layer homogeneously doped with $\mathrm{Si}$, a 20-nm $\mathrm{Al}_{x} \mathrm{Ga}_{1-x}$ As layer, and a 10-nm GaAs cap layer were grown.

[56] O. Göktaş, J. Weber, J. Weis, and K. von Klitzing, Alloyed Ohmic contacts to two-dimensional electron system in Al$\mathrm{GaAs} / \mathrm{GaAs}$ heterostructures down to submicron length scale, Phys. E (Amsterdam, Neth.) 40, 1579 (2008).

[57] Homemade $I / V$ converter with a conversion factor of $4.5 \mu \mathrm{A} / \mathrm{V}$.

[58] A passive low-pass $\pi$ filter of second order is integrated in front of the inputs of the Keithley voltmeter to avoid the noise from the Keithley device down to the Hall bar.

[59] All measured quantities as well as Hall and longitudinal resistance are plotted and checked carefully during the analysis. However, we will present only those diagrams - primarily the longitudinal resistances - that are important for understanding the results. Unless otherwise stated, the remaining plots provide no additional information. In particular, the transition to the breakdown is reversible by tuning the bias voltage, and changing the polarity of the bias leads to the same behavior, as shown in the presented figures.

[60] Low sweep rates avoid eddy currents that would cause huge electrochemical potential differences between the edge and the bulk of the 2DES [70,71]. These nonequilibrium eddy currents affect the breakdown behavior because biased current and eddy currents superpose locally, leading to locally enhanced or reduced Hall fields.

[61] B. E. Kane, D. C. Tsui, and G. Weimann, Evidence for Edge Currents in the Integral Quantum Hall Effect, Phys. Rev. Lett. 59, 1353 (1987).

[62] Owing to the measurement method and numerical calculation of the resistances, the noise level in the resistances is high at small $V$ because the measured voltages and currents are small there and require an exact zero calibration. Our regime of interest lies at larger values of $V$.

[63] S. A. Studenikin, G. Granger, A. Kam, A. S. Sachrajda, Z. R. Wasilewski, and P. J. Poole, Nonlinear magntotransport phenomena in high-mobility two-dimensional electrons in InGaAs/InP and GaAs/AlGaAs, Phys. Rev. B 86, 115309 (2012).

[64] V. Yu, M. Hilke, P. J. Poole, S. Studenikin, and D. G. Austing, Phase diagram of quantum Hall breakdown and nonlinear phenomena for InGaAs/InP quantum wells, Phys. Rev. B 98, 165434 (2018).

[65] The variations in the electron density within the 2DES can occur for various reasons: variations in the donor $D X$ center concentration (within the ItextrmSi-doped $\mathrm{Al}_{x} \mathrm{Ga}_{1-x}$ As layer), the roughness of the interface between the GaAs and the $\mathrm{Al}_{x} \mathrm{Ga}_{1-x} \mathrm{As}$ layer, inhomogeneities in the negative charge distribution present on the heterostructure surface ("Fermi level pinning" at GaAs surfaces), and/or modifications/residuals at the heterostructure surface.

[66] K. Panos, Current distribution and Hall potential landscape within the quantum Hall effect in graphene and towards the breakdown in a $(\mathrm{Al}, \mathrm{Ga}) \mathrm{As}$ heterostructure, Ph.D. thesis, Universität Stuttgart, 2014.

[67] K. Ikushima, H. Sakuma, S. Komiyama, and K. Hirakawa, Imaging of Cyclotron Emission from Edge Channels in Quantum Hall Conductors, Phys. Rev. Lett. 93, 146804 (2004).

[68] K. Ikushima, H. Sakuma, S. Komiyama, and K. Hirakawa, Visualization of quantum Hall edge channels through imaging of terahertz emission, Phys. Rev. B 76, 165323 (2007). 
[69] P. L. McEuen, E. B. Foxman, J. Kinaret, U. Meirav, M. A. Kastner, N. S. Wingreen, and S. J. Wind, Self-consistent addition spectrum of a Coulomb island in the quantum Hall regime, Phys. Rev. B 45, 11419(R) (1992).

[70] T. Klaffs, V. Krupenin, J. Weis, and F. Ahlers, Eddy currents in the integer quantum Hall regime spatially resolved by multiple single-electron transistor electrometers, Phys. E (Amsterdam, Neth.) 22, 737 (2004).

[71] J. Huels, J. Weis, J. Smet, K. v. Klitzing, and Z. R. Wasilewski, Long time relaxation phenomena of a two-dimensional electron system within integer quantum Hall plateau regimes after magnetic field sweeps, Phys. Rev. B 69, 085319 (2004). 